Tesis. Año 12, 11(13), 2018, 31-42

\title{
El rol de los valores éticos/sociales en la in- vestigación científica del embrión humano y la semilla: trigo
}

\author{
Agustín Apaza Yanarico \\ agustinpaza@gmail.com
}

\section{Resumen}

En el presente artículo se sostiene que en cualquier investigación científica de algún fenómeno u objeto natural es difícil evadir de los componentes éticos-sociales en una actividad científica. Se cuestiona la vieja tendencia tradicional de considerar la ciencia libre de valores. Para mostrar que una investigación está impregnada de valores éticos-sociales, usamos como ejemplo dos objetos naturales: el objeto biológico células madre (el embrión) y la semilla agrícola (el trigo). Para desarrollar el trabajo: primero, presentamos el marco conceptual inmerso en el "modelo epistemológico de la interacción ciencia-valores"; segundo, teorizamos una forma de entendimiento de los objetos naturales o fenómenos; luego se muestra que en ellas son indispensables la intromisión de los valores éticos-sociales en la producción del conocimiento científico.

Palabras claves: Ciencia, ética, entendimiento, células madre, semilla.

\begin{abstract}
In the present article it is maintained that in any scientific investigation of some natural phenomenon or object it is difficult to evade the ethical-social components in a scientific activity. It questions the old traditional tendency to consider science values free. To show that an investigation is impregnated with ethical-social values, we use as an example two natural objects: the biological object stem cells (the embryo) and agricultural seed (wheat). To develop the work: first, we present the conceptual framework immersed in the "epistemological model of science-values interaction"; second, we theorize a form of understanding of natural objects or phenomena; then it is shown that in them the intrusion of ethical-social values into the production of scientific knowledge is indispensable.
\end{abstract}

Keywords: Science, ethics, understanding, stem cells, seed. 


\section{El rol de los valores éticos/sociales en la investigación científica del embrión humano y la semilla: trigo}

\section{Introducción}

La filosofía de la ciencia del estilo tradicional, prioritariamente centró su análisis, primero, en el conocimiento científico o específicamente en las teorías; segundo, se ocupó del contexto de justificación, vale decir, de la reconstrucción racional de los resultados científicos más relevantes; tercero, separó tajantemente entre ciencia y valores o entre juicios de hecho y juicios de valor, pregonando la ciencia libre de valores. Hoy en día, ninguna de estas tesis puede ser defendidas, porque empobrecen el panorama temático de la filosofía de la ciencia, reduce el conocimiento a la teoría, la negligencia del papel de los valores éticos-sociales en la producción del conocimiento científico, descuida el rol de las prácticas científicas y la experimentación.

Hoy en día la filosofía de la ciencia vuelve su atención, no solo a los resultados epistémicos, sino, especialmente, al conjunto de la actividad científica: las estrategias metodológicas, la realización de la investigación, la elección de teorías, la diseminación del conocimiento, la aplicación del conocimiento y la evaluación de las consecuencias del conocimiento en la sociedad. Los valores epistémicos y no-epistémicos ${ }^{1}$ tienen roles interconectados en la producción del conocimiento científico.

Para subrayar el papel de los valores éticos-sociales en la producción del conocimiento, abrazamos una corriente filosófica de la ciencia actual que podemos denominar: "modelo epistemológico de la interacción ciencia-valores" (MEIc-v), esta corriente emerge en el propio seno de Círculo de Viena, de la ala izquierda de este grupo, representado por Otto Neurath, Philipp Frank, Edgar Zilsel, entre otros, esta corriente no prosperó debido a la muerte temprana de su líder Neurath y por las persecuciones de la Guerra Fría (Reisch, 2009). La corriente historicista de la filosofía de la ciencia a través de Kuhn trató de reivindicar en los años sesenta, pero este autor fue deshonesto al no hacer ninguna 
mención de referencia; en los años cincuenta hubo un debate intenso sobre el papel de los valores en la ciencia (Rudner, 1953 y Churchman, 1956), Kuhn estaba inmerso en ese mundo, entonces no fue invento del él la reivindicación de los valores en la ciencia como muchos suponen.

Actualmente el MEIc-v, es una corriente exitosa que tiene muchos representes en diferentes partes del globo; renace con fuerza en los años noventa del siglo anterior, entre los representas más ilustres podemos mencionar: Ernan McMullin (1983), Helen Longino (1990), Javier Echeverría (2002), Hugh Lacey (1999, 2010), Heather Douglas, (2009), etc. Se asumirá implícitamente muchas tesis de estos autores para desarrollar nuestra temática del papel de los valores éticos-sociales en la producción del conocimiento científico, especialmente el de Hugh Lacey, quién es el autor más representativo y aplica su epistemología en ciencias más jóvenes como las ciencias agrícolas, que no es común en la filosofía de la ciencia tomar como referencia a las ciencias como la farmacéutica, pecuaria, alimentaria, medio ambiental, energética, etc., sino a las ciencias más tradicionales como la física, la química o la biología. El marco conceptual del MEIc-v permite la evaluación de estas nuevas ciencias jóvenes de forma exitosa epistemológicamente.

Para abordar el tema, primero, se presenta el marco conceptual del MEIc-v, a fin de contextualizar cómo entran los valores éticos-sociales en el desarrollo de una investigación científica. Se cuestiona la postura tradicional de la ciencia libre de valores, fundamentado apenas en dos contextos: justificación y descubrimiento. Desde el MEIc-v se identifica varios contextos o momentos en el desarrollo de una actividad científica, se enfatiza que los valores éticos-sociales tienen papeles legítimos en la producción del conocimiento científico.

Segundo, se muestra con dos ejemplos de investigación científica: células madre (embrión) y la semilla (trigo); cómo en el análisis de estos objetos naturales los intereses valorativos epistémicos y no-epistémicos, determinan dialécticamente el tipo de conocimiento contextual a ser producido.

\section{Modelo epistemológico de la interacción ciencia-valores (MEIc-v)}

Desde el MEIc-v se entiende, que en la gran parte de la tradición de la ciencia moderna se sostiene que la ciencia es libre de valores, esta idea se concibe mejor en la combinación de tres tesis o conceptos: imparcialidad, neutralidad y autonomía, estos términos son los valores constitutivos de las prácticas científicas y de las correspondientes instituciones científicas (Lacey, 1999).

La tesis de la imparcialidad presupone una distinción entre valores epistémicos y no-epistémicos, se afirma que una teoría es adecuadamente aceptada si y solo si, ella manifiesta los valores cognitivos en un alto grado en la luz de 
los datos empíricos relevantes. Los valores morales y sociales nada tendrían que ver con la aceptabilidad de una teoría. El MEIc-v concuerda con esta tesis sin ambigüedad.

La tesis de la neutralidad sostiene que los resultados científicos pertenecen al patrimonio común de la humanidad y sirven equitativamente a todas las perspectivas de valor, niega cualquier papel de los valores éticos y sociales en una actividad científica. Esta tesis está comprometida actualmente con las prácticas científicas mercantilistas y dominantes. Pero sería loable si se manifestase en las investigaciones científicas dentro de un pluralismo de metodologías (Lacey, 2010).

La tesis de la autonomía sostiene que las prácticas científicas son guiadas con el objetivo de obtener teorías según los requisitos de la imparcialidad y neutralidad, por tanto, ellas proceden mejor si no están sujetas a influencias externas. En la actualidad, se usa el término autonomía en las prácticas científicas dominantes con el fin de protegerse de las supuestas intromisiones valorativas externas en la investigación.

El ideal de la autonomía y la neutralidad son interesantes en las prácticas científicas, pero en los hechos, estos ideales no son sustentables actualmente, puesto que las investigaciones científicas se realizan de acuerdo a los intereses del mercado. El desafío hoy está en reivindicar una autonomía-calificada en donde las instituciones científicas mantengan diálogos con una amplia variedad de grupos sociales que incorporen diferentes perspectivas de valor. Una autonomía-calificada, podría contribuir para una manifestación más alta de la neutralidad.

Desde el MEIc-v, también se puede afirmar, que cualquier investigación científica siempre es conducida bajo una estrategia cuyos roles principales son: primero, restringir los tipos de teorías (o hipótesis) a ser considerados y posiblemente confirmados en un proyecto de investigación que lleva hacia una especificación de los recursos conceptuales disponibles de los tipos de posibilidades que pueden ser identificados, sus modelos, analogías y estimulaciones que pueden ser usados en el curso de la investigación; segundo, seleccionar los tipos de datos empíricos que se buscan y se relatan, y cuáles de los fenómenos y aspectos que pueden ser observados e investigados (Ibíd.).

La ciencia moderna ha sido desarrollada, por lo general y casi siempre, exclusivamente bajo una estrategia (con algunas variedades) denominada: estrategias de enfoque descontextualizante (EED), bajo las cuales las teorías se restringen para representar los fenómenos y las encuadran sus posibilidades generativas a partir de las estructuras subyacentes (y sus componentes), el proceso, la interacción y las leyes que los gobiernan, expresados típicamente en términos matemáticos. Podemos observar que la representación de los fenómenos de esa 
forma envuelve su descontextualización, vale decir, los fenómenos están disociados de cualquier de las relaciones que puedan tener con los acuerdos sociales, con las cualidades sensibles y los valores, de cualquiera de las posibilidades que ellos puedan obtener en virtud de su inserción en contextos particulares de lo social, lo humano y lo ecológico. Los datos empíricos son presentados usando categorías descriptivas que generalmente son cuantitativas y aplicables en virtud de sus mediciones, uso de instrumentos y operaciones experimentales (Ibíd.).

Desde el MEIc-v se observa que no todos los fenómenos u objetos de investigación científica pueden ser investigados adecuadamente bajo el enfoque descontextualizante. La investigación empírica sistemática que se produce de acuerdo con la imparcialidad, pueden y precisan ser conducidas bajo otras estrategias alternativas no necesariamente reducidas a estrategias de enfoque descontextualizante, como se mostrará más adelante en la investigación de células madre y la semilla. La adopción del pluralismo metodológico hace posible que todos los fenómenos pueden, en principio, ser investigados con otras metodologías alternativas no necesariamente reducidas a la EED.

Lógicamente, existen muchas razones por la simpatía exclusiva por la EED de la ciencia moderna: (i) por la fecundidad de producir los resultados científicos de acuerdo con la imparcialidad y la potencialidad del desarrollo prácticamente ilimitado; (ii) se adopta la EED porque está relacionado mutuamente reforzadas con los valores del progreso tecnológico de dominación y, (iii) los valores del progreso tecnológico están inmersas en todos los países industrialmente avanzados, incluso incorporados en sus instituciones más importantes. Los países periféricos están presos a esos modelos, no ven alternativas de desarrollo. Entonces, existen buenas razones para el privilegio de asumir las EED, en la medida en que hay buenas razones para sustentar los valores del progreso tecnológico (Ibíd.).

Cabe observar, que los valores sociales pueden promover fuertes razones para adoptar un tipo de estrategia específico, como muestra el ítem (i) arriba sobre la fecundidad de la EED, relacionado con el ítem (ii) de la adopción mutuamente reforzadora de los valores del progreso tecnológico, por ejemplo, la dominación de la naturaleza para extraer los recursos naturales de forma salvaje en nombre del progreso económico y social, sin ningún respeto al medio ambiente. Vale decir, por más que la EED niegue la intromisión de los valores éticos y sociales en una investigación científica, ella misma incurre inmersa en reivindicar una investigación en nombre de los valores del progreso tecnológico. Parece no haber espacio para negar los valores éticos sociales en una investigación científica.

En la tradición de la ciencia moderna con estrategia neopositivista consideró, y aún considera, que en una actividad científica solamente existen dos 
contextos: contexto de descubrimiento y contexto de justificación, el primero, incumbe apenas con las cuestiones volitivas y nada tendría que ver con las cuestiones epistemológicas, puesto que ella es subjetiva; el segundo, sería un asunto típicamente epistemológico, en la medida que sólo se ocupa de la construcción del contexto justificativo. En otras palabras, esta vieja doctrina afirma que los valores éticos y sociales no pueden tomar parte de la producción del conocimiento científico, solo compete al segundo contexto, por tanto, la ciencia sería libre de valores. Además, esta propuesta sugiere directamente una separación entre juicios de hecho y juicios de valor, tan cuestionado en nuestros días. Actualmente, sustentar esta vieja postura implica concebir la ciencia de forma obliterada, aún más, reduce el análisis de la ciencia solo a la teoría como producto (Echeverría, 2002).

Desde el MEIc-v, podemos concebir la ciencia de forma más adecuada desde los varios contextos que involucran una práctica científica. Se pueden identificar y distinguir por lo menos seis contextos o momentos (M) interconectados en una actividad científica: $\mathbf{M}_{\mathbf{1}}$ la adopción de una estrategia metodológica para la investigación, $\mathbf{M}_{2}$ la realización de la investigación, $\mathbf{M}_{3}$ la evaluación cognitiva de las teorías e hipótesis como portadoras del conocimiento y entendimiento, $\mathbf{M}_{4}$ la diseminación de los resultados científicos, $\mathbf{M}_{5}$ la aplicación del conocimiento científico y, $\mathbf{M}_{6}$ las implicaciones del conocimiento en la sociedad. En todos estos momentos los valores éticos-sociales tienen papeles legítimos en la producción del conocimiento, apenas en el $\mathrm{M}_{3}$ se hace un paréntesis, ya que en ella cabe solo a los valores epistémicos en la elección de una buena teoría científica imparcialmente, se deja de lado el factor valorativo (Lacey \& Mariconda, 2014). Como podemos observar la consideración de estos varios contextos en la actividad científica, no deslegitima el papel de los valores éticos y sociales en la producción del conocimiento científico. Desde esta postura se refuta la vieja tesis de la ciencia libre de valores. Se asume que la ciencia está impregnada de valores, no hay lugar para afirmar sobre la supuesta autonomía y neutralidad de la ciencia; se sostiene que cualquier emprendimiento científico depende de las condiciones económicas en donde se desarrolla. La misma afirmación: la ciencia es libre de valores, es una afirmación valorativa.

En el marco del MEIc-v, el objetivo o los fines de la ciencia, no solo está pensado para obtener el entendimiento de los fenómenos de acuerdo con la imparcialidad, sino también tal entendimiento puede ser usado para ampliar nuestros poderes sobre el control de los objetos de forma sustentable para el crecimiento económico, como también para elaborar buenas políticas públicas y los reglamentos para regular de forma apropiada en la implementación de las innovaciones tecnocientíficas. En tal sentido, desde el MEIc-v se puede especificar los siguientes fines: 
(i) generar y consolidar el conocimiento y entendimiento de los fenómenos del mundo, de sus posibilidades (incluso de los fenómenos generados en espacios experimentales y en las prácticas tecnológicas):

- de ámbitos crecientemente mayores de fenómenos, incluso de los fenómenos producidos o propuestos en el curso de las operaciones experimentales y de medida (frecuentemente con el objetivo de testear teorías o de informar innovaciones tecnocientíficas).

- ningún fenómeno de importancia o de valor en la experiencia humana o en la vida de la práctica social (y de las hipótesis sobre ellas - incluso de los efectos colaterales y riesgos de las implementaciones en las aplicaciones prácticas) en principio pueden ser excluidos del alcance de la investigación científica. Y consecuentemente:

(ii) hacer descubrimientos de los nuevos fenómenos y de las nuevas maneras de generar fenómenos; anticipar las posibilidades que pueden originarse en las consecuencias causales de los fenómenos; $y$, a veces, hacer previsiones de los fenómenos futuros.

(iii) usar el entendimiento obtenido para informar las actividades prácticas, incluso aquellas basados en la implementación de las innovaciones tecnocientíficas $\mathrm{y}$,

- teniendo en vista la aplicación práctica y tecnológica del conocimiento, de los descubrimientos, de las anticipaciones (y, a veces, la investigación está conducida directamente para producir ellas) - en el contexto del horizonte proporcionado por el pluralismo metodológico.

- El valor de obtener el entendimiento sobre los fenómenos del mundo o de algún objeto natural está subordinado a la expansión de nuestro conocimiento acerca de qué podemos hacer, de cómo podemos ampliar nuestros poderes con el fin ejercer el control sobre los objetos de forma sustentable, especialmente en la medida que pueden contribuir para el crecimiento económico; esta misma estrategia puede ser aplicado a los intereses de las principales corporaciones comerciales del tipo tecnocientífico, pero tomando en cuenta la cláusula de sustentabilidad.

(iv) obtener el conocimiento y buscar los datos empíricos, que sean apropiados para las deliberaciones sobre la legitimidad de las aplicaciones del $\mathrm{co}^{-}$ nocimiento científico y para la formación de las políticas públicas y de los reglamentos, que precisan acompañar las implementaciones de las innovaciones tecnocientíficas, de modo que los resultados de la investigación sean evaluados (por su valor cognitivo) en el contexto de una perspectiva encuadrada por el ideal de la imparcialidad. 
- las reivindicaciones de los fenómenos hacen parte del acervo del conocimiento cientifico establecido, solamente cuando son incorporadas en teorías aceptadas de acuerdo con la imparcialidad (las hipótesis que informan las innovaciones tecnocientíficas y las que explican su eficacia son generalmente de este tipo)

- las reivindicaciones que tienen más o menos apoyo empírico, endosadas para el fin de afirmar acciones, actividades prácticas, políticas públicas y reglamentos no deben ser distinguidas del conocimiento aceptado de acuerdo con la imparcialidad; y, los endosamientos no deben ser hechos en desacuerdo con las propuestas aceptadas de acuerdo con la imparcialidad, deben estar basadas en datos empíricos obtenidos y en investigaciones que no impidan la posibilidad eventual (posterior de más investigación, si condujese) de obtener resultados que concuerden con la imparcialidad (Apaza, 2013).

Estos son algunos de los conceptos fundamentales del MEIc-v para comprender cómo los valores epistémicos y no-epistémicos entran dialécticamente en el entendimiento y conocimiento de los objetos naturales o de algún fenómeno.

\section{Entendimiento científico de los objetos naturales: el embrión y la semilla}

El entendimiento de cualquier fenómeno u objeto científico, por lo general, pueden generar las siguientes preguntas: ¿Qué son los objetos y los fenómenos naturales? y ¿en qué consiste el entendimiento de ellas? Las respuestas pueden ser diversas, de acuerdo al paradigma científico que uno puede estar incluido. En tal sentido, el entendimiento de ellas es siempre contextual y el propio concepto del entendimiento varía de acuerdo con el contexto, según el enfoque de interés y con los agentes del discurso. Lo que siempre hace parte del entendimiento de una cosa, por lo general, implica los siguientes componentes:

- Una afirmación con respecto de qué es: el tipo de cosa que ella es, sus propiedades, sus comportamientos, sus relaciones y sus variaciones temporales.

- Una afirmación respecto de por qué una cosa es como es.

- Una afirmación respecto de sus posibilidades: cuáles son las posibilidades que están abiertas (incluso las posibilidades no realizadas hasta el momento, considerando sus propios poderes para desarrollar y sus interacciones con las otras cosas).

Entonces, el entendimiento de un fenómeno u objeto natural, parece requerir siempre afirmaciones del tipo: "qué es", "por qué es" y "lo que es posible". A 
cada una de estos tres componentes se pueden atribuir varias interpretaciones. Con respecto al "qué es" un objeto, se puede referir a un objeto de nuestra experiencia, a un objeto de una práctica humana, o al componente de una estructura, etc., (por ejemplo, un sistema ecológico, social o cósmico; en nuestro caso una semilla o el embrión humano); son objetos que mantiene relaciones causales con otros objetos.

Dependiendo de cómo los objetos son considerados, las respuestas a las preguntas “¿por qué es?” y “¿qué es posible?”, podría tomar formas diferentes y reflejar intereses diferentes. Por ejemplo, una semilla: el trigo y las células madre: el embrión. Estas entidades pueden suscitar la pregunta: ¿Cuáles son las posibilidades que le están abiertas? La semilla puede ser considerada de muchas maneras: primero, puede ser un objeto que genera, después del cultivo, cosechas cuantificables en rendimientos; segundo, puede ser un objeto que participa integralmente de procesos sociales, un símbolo de libertad. Las células madre embrionarias son considerados para algunos en sus primeras fases como un amontonado de células disponibles para la manipulación terapéutica regenerativa; para otros, desde que se unen los dos gametos femenino y masculino, tornándose en un embrión, son personas o seres humanos, por tanto, inviolables, tanto en el vientre de la madre como en el desarrollo in vitro.

En el ejemplo de las células madre embrionarias, para unos, la entidad embrionaria de las primeras fases no posee el ser de la condición humana hasta bien avanzado del proceso de la organogénesis, prácticamente hasta que el desarrollo del sistema nervioso central se haga objetivable en algún nivel de actividad psicológica o de autoconciencia. Pues en tanto no se manifieste signos de un incipiente psiquismo, no podría afirmarse que el embrión sea constitutivamente humano. La afirmación de lo humano exigiría, pues, de la objetivación del psiquismo como antecedente de la racionalidad. Solo a partir de entonces, vale decir, a partir de una mínima expresión de psiquismo daría condición a la consideración de un ser humano. El proceso de la humanización del embrión pasaría por un desarrollo gradual. La suficiencia constitucional, el aflorar de todas, de todos los ítems humanos, no estaría presente hasta que no dieran evidencias de actividad de psiquismo, el pre-embrión o el embrión preimplantatorio y sus etapas ulteriores, previas a las evidencias del psiquismo, no justificaría hablar de vida humana en un sentido estricto, tampoco de una personeidad en la interpretación filosófica, ni obviamente de dignidad o de respeto (De Santiago, 2004). Entonces, los embriones preimplantatorios no serían sino un amontonado de células, permisibles para la experimentación tanto para la investigación básica como para la medicina regenerativa para los propios órganos del cuerpo humano; además detrás de esta concepción de células preimplantatorios corren grandes intereses de corporaciones comerciales para su justificación y comercialización en nombre del progreso tecnológico. 
Para otros, desde el punto de vista biológico, filosófico y antropológico, el embrión humano desde la constitución del zigoto es un ser humano, un ser viviente. $\mathrm{El}$ hombre desde su concepción no es un algo, sino un alguien que tiene un carácter personal. Vale decir, las características biológicas del zigoto y del embrión preimplantatorio permitirían considerarlo como un organismo incipiente, como un individuo de la especie humana que inicia su singularidad existencial mediante el despliegue de consecutivos fenotipos, que expresan en cada tiempo la información codificada de la que es dueño y portador. Entonces, la información de esta actividad de despliegue y desarrollo, sería la que determina la vida biológica y su potencial de desarrollo ulterior. Por tanto, se afirma de él como una persona en cuanto personeidad, porque la suficiencia constitucional se produce o se cristaliza en el nivel del zigoto. Desde este punto de vista, el embrión humano no puede ser sujeto de experimentaciones tecnocientíficas, es persona desde su primera concepción (López-Moratalla, 2004, 2006 y López-Moratalla et al, 2011).

En el caso de las semillas, por un lado, pueden ser alteradas bajo el efecto de las nuevas tecnologías genéticas y tornarlos "híbridas", de tal forma que, cuando son cultivadas bajo ciertas condiciones específicas, los rendimientos de las cosechas pueden aumentar significativamente. Por otro lado, después de tornarse generadoras de los referidos aumentos de rendimientos, las semillas pueden transformarse en una mercadería comercial-social (un objeto producido y cultivado intensamente por empresas capitalistas y comercializadas según las reglas del mercado). Entonces, la semilla como objeto, que en la mayoría de las veces era producido en cosechas anuales como parte de las costumbres locales para el alimento, pero también cumplía una función social, representar el símbolo de la vida (Shiva, 2001 y Lacey, 2006). Así, la semilla como objeto va teniendo otras relaciones de orden social según las diferentes prácticas humanas. Vale decir, la semilla bajo la intervención de la tecnociencia pasa a tener un aumento cuantitativo, sufre un cambio social fundamental, deja de ser visto apenas como una cadena de alimentar o un símbolo social, sino pasa a ser visto para los agentes del capitalismo como un objeto mercantil. Esto implica que un objeto va cobrando diferentes formas de entendimiento, dependiendo de los intereses valorativos de las prácticas sociales, en vista de ello se puede sostener que el entendimiento es contextual y el conocimiento obtenido cae en lo mismo.

Es interesante notar que no hay acuerdo entre los propios científicos sobre la interpretación de la entidad biológica: el embrión y la entidad agrícola: la semilla; cada científico interpreta los objetos de investigación de acuerdo al paradigma en donde está envuelto. Por ejemplo, en el caso de la semilla, los que ven este objeto mercantilmente son los científicos formados en la literatura transgénica; mientras los científicos con formación agroecológica lo ven como objetos renovables, aún no necesarios para la intervención tecnocientífica. En 
el caso del embrión, los que ven como un amontonado de células, factibles para su manipulación terapéutica y su comercialización, son por lo general los que abrazan el paradigma de la ética principialista; en cambio los que se oponen y consideran el embrión como un ser humano desde la primera concepción, pertenecen al paradigma de la ética personalista. Como podemos notar los valores ético-sociales tienen papeles legítimos y fundamentales en el entendimiento e interpretación de los objetos naturales; no son suficientes los criterios epistémicos para producir el conocimiento científico, inevitablemente existen intereses de perspectivas de valores sociales en la producción de un conocimiento, aún más, de forma contextualizada.

\section{Conclusiones}

En gran medida, parece que el entendimiento de cualquier fenómeno u objeto natural implica siempre estar inmerso en alguna estrategia metodológica o paradigma, vale decir, una comunidad científica tiene o adopta sus propios criterios epistémico y no-epistémicos para entender alguna parcela de la realidad o alguna entidad natural. Como se mostró con los ejemplos de la semilla y el embrión, los propios científicos al investigar el mismo objeto experimentalmente discuerdan en su interpretación, ello se debe por lo general a los propios intereses que corren detrás de la investigación, por tanto, los valores éticos-sociales juegan papeles determinantes en la producción del conocimiento, esto no es el ideal de conocer el mundo por el amor del conocimiento y de forma sustentable; sino lamentablemente la educación del sistema social capitalista demanda así, el problema es cómo evitar ello, esta tarea queda pendiente para los investigadores honestos y comprometidos con una investigación sustentable para producir el bienestar social de todos de forma equitativa.

\section{Nota}

1 Con los términos "valores epistémicos" nos referimos a los valores que justifican una buena teoría científica: la coherencia intertónica, la simplicidad, la exactitud, la adecuación empírica, la verdad, etc. Por "valores no-epistémicos" nos referimos a los valores éticos y sociales que tienen papeles legítimos en la producción del conocimiento: la sustentabilidad, el bienestar social, la utilidad, etc.

\section{Referencias}

Apaza, Agustín. (2013). Las prácticas científicas y los valores: una introducción a la filosofia de la ciencia de Hugh Lacey (Tesis de Magister). Lima: Universidad Nacional Mayor de San Marcos.

Churchman, West. (1956). Science and decision making. Philosophy of Science, 23(3), 247-249. 
Douglas, Header. (2009). Science, policy, and the value-free ideal. Pittsburgh: University of Pittsburgh Press.

Echeverría, Javier. (2002). Ciencia y valores. Barcelona: Ediciones Destino.

Lacey, Hugh. (1999). Is science value free? Values and scientific understanding. London: Routledge.

-. (2006). La controvérsia sobre os transgênicos. Questões cientificas e éticas. São Paulo: Ideias \& Letras

-. (2010). Valores e atividade científica 2. São Paulo: Editora 34 y Associação Filosófica Scientiae Studia.

Lacey, Hugh \& Mariconda, Pablo. (2014). O modelo da interação entre as atividades científicas e os valores na interpretação das práticas científicas contemporâneas. Estudos Avançados, 28(82), 181-199.

Longino, Helen. (1990). Science as social knowledge. Princeton (NJ): Princeton University Press.

López-Moratalla, Natalia. (2004). La realidad del embrión humano en los primeros quince días de vida. Persona y Bioética, 7-8, 6-23.

- (2006). Racionalidad de la investigación con células troncales embrionarias. Cuadernos de Bioética, XVII, 327-347.

López-Moratalla, Natalia; Santiago, Esteban \& Herrans, Gonzalo. (2011). Inicio de la vida de cada ser humano ¿Qué hace humano el cuerpo del hombre? Cuadernos de Bioética, XXII, 283-308.

Mcmullin, Ernan (1983). Values in science. En: Asquit, P. \& Nickles, T. (eds.) PSA. East Lansing: Philosophy of Science Association, p. 3-28.

Reisch, George. (2009). Cómo la Guerra Fría transformó la filosofía de la ciencia. Hacia las heladas laderas de la lógica. Bernal: Universidad Nacional de Quilmes.

Rudner, Richard. (1953). The scientist qua scientist makes value judgments. Philosophy of Science, 20(1), 1-6.

Santiago, Manuel De. (2004). Estatuto biológico, antropológico y ético del embrión humano. Bioética Web. Recuperado de: http://www.bioeticaweb.com/ estatuto-biolasgico-antropolasgico-y-atico-del-embriasn-humano-dr-manuel-de-santiago/ Accedido el: 02/06/2017.

Shiva, Vandana. (2001). Biopirataria: a pilhagem da natureza e do conhecimento. Petrópolis RJ: Vozes. 